\title{
Active Distribution Network Bifurcation and Voltage Stability Research
}

\author{
Xianbin Dai \\ Electrical Engineering Department, Shenyang Institute of Engineering, \\ China ,dxbcy@126.com
}

Key words: bifurcation theory, active distribution network, voltage stability

\begin{abstract}
It analyzes the common bifurcation categories in active distribution network; based on the active distribution network's wind field system model, it changes the reactive power parameters of its load model and conducts studies on the influence brought by reactive power on voltage stability based on bifurcation theory; it calculates Hopf Bifurcation Point in the wind power system containing SVC; it judges Hopf Bifurcation based on parsing algorithm; it analyzes the influence brought by reactive power and VC on wind power system. The result indicates that the increase of reactive power in wind power system will create Hopf Bifurcation in the system and it will use SVC to conduct reactive compensation, by which it will delay Hopf Bifurcation and elevate the active distribution network's voltage stability.
\end{abstract}

\section{Introduction}

Active distribution network is a kind of development pattern for future intelligent distribution network in China. ${ }^{[1]}$ GRE C6.11 Workgroup defines active distribution network as "distribution network that is able to comprehensively control distributed power (DG, flexible loading and storage) and it is able to use the flexible network technology to realize effective tide management and distributed power undertakes the role as support for the system under the reasonable surveillance environment and access code." It aims at enhancing distribution network's ability of accepting renewable energies, elevating the use rate of assets in distribution network, delaying upgrading investment on distribution network, optimizing primary energy structure and improving users' power use quality and power supply reliability. Active distribution network is able to combine and control various distributed energies (DG, controllable load, storage and management at the demand side, etc). Nevertheless the access of distributed energies in large amount makes its operation approach its limit, by which reactive power and voltage stability are facing severe challenges and problems related to voltage stability might happen at any time. ${ }^{[2]}$

Bifurcation Theory is an important branch in non-lineal science and it mainly studies the change process of both structure of solution and stability caused by the change of parameters in system. Therefore, bifurcation theory is an effective tool used to analyze the structural stability in non-lineal dynamic system. Active distribution network is a complicated non-lineal dynamic system and the change of voltage stability is actually the process converting from stable status to bifurcation. When parameters of active distribution network change within a certain range, the variables of state corresponding to the parameters change within a stable domain of attraction; when the parameters remain on the boundary of this range, the structure in the distribution network will approach status of bifurcation; when parameters cross the boundary value, bifurcation happens to the distribution network. Practice indicates that reasonable distribution (reactive optimization) of reactive power is the most effective method of guaranteeing voltage stability. ${ }^{[3]}$ Establish the mathematical model of active distribution network based on bifurcation theory and conduct reactive power's optimization study, which contains the great significance of deeply understanding the dynamic essence of active distribution network and the influence on voltage stability brought by reactive distribution. 


\section{General Bifurcation Types and Characteristics in Active Distribution Network}

Active distribution network belongs to the non-lineal system operating close to its limit status with large capacity and multiple connections and it is able to describe it with a set of differential algebraic equations. Therefore, bifurcation theory and study on voltage stability in electric power system must have the necessary connection. Diversified bifurcation phenomena exist in active distribution network but voltage instability and bifurcation phenomena are complicated and diversified. Generally speaking, there are two forms of bifurcation - Saddle-node Bifurcation and Hopf Bifurcation. Sometimes bifurcation directly leads to system voltage instability and sometimes it deteriorates system static characteristics.

A simple active distribution network can be described by differential algebraic equation (1): in the equation, $f$ is the differential algebraic equation used to describe dynamic behaviors of power generator and loads in the distribution network; $g$ is the algebraic equation used to describe active and reactive power balance in each node; $\mathrm{x}$ is system status variable; $\mathrm{y}$ is system algebraic status variable; $\mu$ is system control parameter;

$$
\left\{\begin{array}{l}
\dot{x}=f(x, y, \mu) \\
0=g(x, y, \mu)
\end{array}\right.
$$

Conduct differential transformation in equation (1) at bifurcation point $\left(\mathrm{x}_{0}, \mathrm{y}_{0}\right)$, we will obtain differential equation (2) of dynamic characteristics in active distribution network:

in the equation,

$$
\left\{\begin{array}{l}
A=D_{x} f\left(x_{0} y_{0}\right), B=D_{y} f\left(x_{0} y_{0}\right), C=D_{x} g\left(x_{0} y_{0}\right), D=D_{y} g\left(x_{0} y_{0}\right), J=\left[\begin{array}{ll}
A & B \\
C & D
\end{array}\right] . \\
\frac{d x}{d t}=\left(A-B D^{-1} C\right) x=J x
\end{array}\right.
$$

System's dynamic characteristics can be fully determined by characteristic value of Jacobian Matrix J. According to bifurcation theory, it uses change of characteristic value of Jacobian Matrix $\mathrm{J}$ based on the change of control parameters to identify different bifurcation points.

Saddle-node Bifurcation - if the active distribution network described in Equation (1) meets with the following condition, system bifurcation point $\left(\mathrm{x}_{0}, \mathrm{y}_{0}\right)$ becomes one saddle point and one node and this kind of bifurcation is called Saddle-node Bifurcation. ${ }^{[4]}$

$$
\left\{\begin{array}{l}
f\left(x_{0} y_{0} \mu_{0}\right)=0 \\
\operatorname{ran}\left[D_{x} f\left(x_{0} \mu_{0}\right)\right]=n-1 \\
\operatorname{ran}\left[D_{y} f\left(y_{0} \mu_{0}\right)\right]=n-1 \\
\operatorname{ran}\left[D_{x} f\left(x_{0} \mu_{0}\right), D_{y} f\left(y_{0} \mu_{0}\right), D_{\mu} f\left(x_{0} \mu_{0}\right), D_{\mu} f\left(y_{0} \mu_{0}\right)\right]=n
\end{array}\right.
$$

Saddle-node Bifurcation is the most common partial static bifurcation in active distribution network. It is the bifurcation for the disappearance of balance point in vector field and its bifurcation phenomenon normally contains linearization matrix with determinant at zero in the balance point of vector field. In the analysis of practical electric power system failures, the use of filtering equipment will detect that most voltage instability phenomena are connected with saddle-node bifurcation. Monotonic voltage instability in electric power system is related to saddle-node bifurcation. When saddle-node bifurcation occurs, the corresponding Jacobian Matrix J has one zero characteristic value.

Hopf Bifurcation , if $\mu=\mu_{0}$, system will create non-hyperbola equilibrium point $\left(\mathrm{x}_{0}, \mathrm{y}_{0}\right)$ and the corresponding Jacobian Matrix J contains the characteristic value of zero real part. In the condition, any minor disturbance will cause change to structural stability in the system and it will cause dynamic bifurcation at balance point $\left(\mathrm{x}_{0}, \mathrm{y}_{0}\right)$. If active distribution network described in Equation (1) meets with the following condition, the system's bifurcation point $\left(\mathrm{x}_{0}, \mathrm{y}_{0}\right)$ is called Hopf Bifurcation. 


$$
\left\{\begin{array}{l}
f\left(x_{0} y_{0} \mu_{0}\right)=0 \\
D_{x} f\left(x_{0} \mu_{0}\right), D_{y} f\left(y_{0} \mu_{0}\right), D_{\mu} f\left(x_{0} y_{0} \mu_{0}\right) \\
d\left[\operatorname{Re} \lambda\left(\mu_{0}\right)\right] / d \mu \neq 0
\end{array}\right.
$$

When Hopf Bifurcation occurs, the characteristic value of the balance point (bifurcation point) corresponding to dynamic system has a pair of conjugate pure virtual roots. Hopf Bifurcation is related to oscillation instability of active distribution network system. When supercritical Hopf Bifurcation occurs to the system, one stable limit loop occurs at the bifurcation point and one stable balance point becomes instable with oscillation that keeps increasing and finally gets attracted by one stable limit loop. When subcritical Hopf Bifurcation occurs to the system, one instable limit loop (it exits before bifurcation occurs) is contracted and finally disappears when the bifurcation point combines with one stable balance point. After bifurcation occurs, the balance point becomes instable to cause the continuously rising oscillation.

\section{Bifurcation Simulation Study}

Active distribution network's system model is as illustrated in Fig.1. ${ }^{[5]}$ The model is composed of two generators and one wind power field. G1 is infinite power, G2 is classic synch power generator model, G3 is wind power plant model, dynamic load adopts the asynchronous generator with equivalent value, SVC is static reactive generator and the dynamic differential equation set used to describe this model system is illustrated in Equation (1).

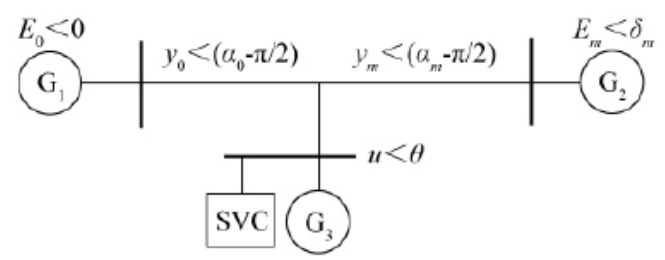

Fig.1. Active Distribution Network's System Model

Saddle-node Bifurcation, control parameter $\mathrm{q}=11.3$, initial condition for calculation: $\mathrm{x}, \mathrm{y}$ $=(\delta, \omega, \theta, \mu)=(0.28,0,0.18,0.92)$. As what is illustrated in Fig.2 and Fig.3, in the above-mentioned saddle-node bifurcation point, when $\mathrm{t}=0$, reactive power increases from $1.242539 \mathrm{p} . \mathrm{u}$ to 1.442539p.u., wind field's bus voltage V rapidly drops to about 0.73p.u. Once the saddle-node bifurcation occurs, wind field will get minor disturbance and the bus voltage will present the monotonic drop at large amplitude.

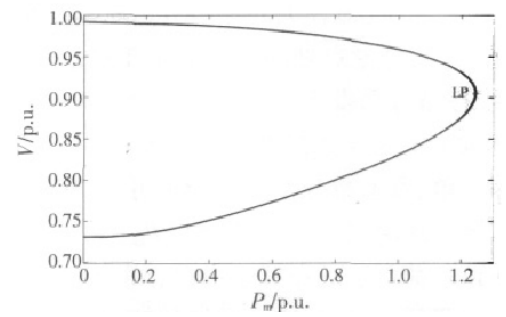

Fig.2. Wind Terminal Voltage Change Curve along with Reactive Loads

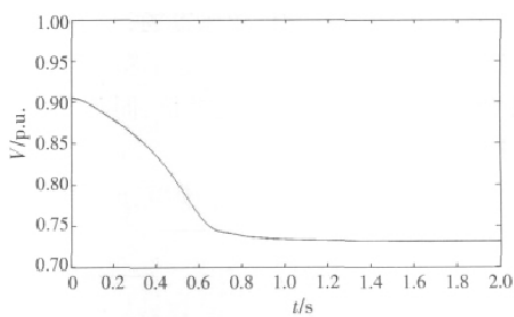

Fig.3. Wind Terminal Voltage Change Curve along with Time

When wind field system is operation in heavy load, the injection power to wind power field at the saddle-node bifurcation point is small, namely, system's ability to undertake wind power change 
and to maintain voltage stability is vulnerable. In order to delay the happening of saddle-node bifurcation of electric power system when it is operation in heavy load, it shall use SVC to replace the parallel capacitor sets to conduct reactive compensation for asynchronous generator terminal.

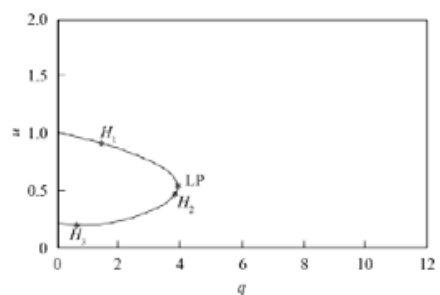

(a)

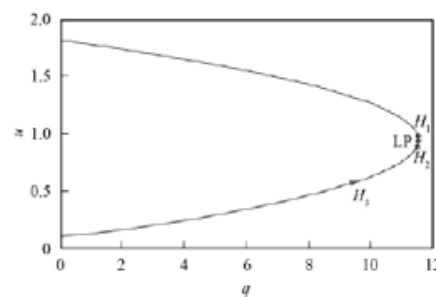

(b)

Fig.4. Wind Field Terminal Voltage Change Curve along with Reactive Load

Hopf Bifurcation, control parameter $\mathrm{q}=10.4$, initial condition for calculation: $\mathrm{x}, \mathrm{y}=(\delta, \omega, \theta, \mu)$ $=(0.2,0.45,0.12,0.9)$, system balance point (bifurcation point) is $(0.301,0,0.121,1.141)$.

Fig. 4 is bifurcation curve of wind filed voltage $u$ along with the change of reactive power $q$. when active power output by asynchronous power generator gradually increases, the reactive power q it absorbs will also gradually increase. Based on Fig.4 (a), we can see that the increase of reactive power will lead to the drop of voltage $\mathrm{u}$ in wind field and when system approaches the limit point LP, namely, $q=1.395$, system voltage $\mu=0.893$, and Hopf Bifurcation occurs to wind power system. Based on the analysis algorithm given in this paper, it is able to obtain one pair of conjugate virtual roots $\lambda \mu \mathrm{c}= \pm \mathrm{j} 6.8961$, characteristic root of wind power system and system curvature factor $\beta=0.0301$. According to Hopf Bifurcation theory, subcritical bifurcation occurs to the system. When subcritical Hopfs Bifurcation occurs to the wind power system, asymptotic stability of jumps near the bifurcation point will be converted to instable non-lineal oscillation. Therefore reactive power absorbed by wind field will influence system voltage stability.

Fig.4 (b) is wind field terminal voltage's Hopf Bifurcation Curve when SVC participates in reactive compensation $\mathrm{Kr}=1.43$, $\mathrm{Tr}=0.015$, Based on Fig.4 (b), when SVC is installed, it improves the voltage level in wind power system, increases the reactive power ( $q=11.53$ ) at H1 Point of Hopf Bifurcation and H1 Point infinitely approaches LP Point. Simulation result indicates that SVC uses the reactive power absorbed by wind field to delay Hopf Bifurcation point to effectively improve system voltage's stable operation domain. Nevertheless, SVC is unable to get rid of Hopf Bifurcation point to enable system safely operate before the limit point.

\section{Conclusion}

Simulation result indicates that when saddle-node bifurcation occurs and wind field suffers from minor disturbance, the bus voltage will create monotonic drop with large amplitude. When subcritical Hopf Bifurcation occurs to the wind field, in the area near the bifurcation point, the asymptotic stability of jumps will be converted to instable non-linear oscillation. The reactive power absorbed by wind field will influence voltage stability in electric power system. The use of SVC will compensate for reactive power absorbed by wind field, delays Hopf Bifurcation point and effectively improve voltage's stable operation domain. Nevertheless, SVC is unable to get rid of Hopf Bifurcation point to enable system safely operate before the limit point.

\section{Acknowledgements}

The paper is sponsored by "SIE Technology Fund - General Project (LGYB-1414)"

\section{References}

[1] Yi You, Dong Liu, Wenpeng Yu, Active Distribution Network Technology and Development, Electric Power System Automation, Vol.18 (2012), p.10-16. 
[2] Chengshan Wang, Peng Li, Development Challenges of Distributed Generation, the Micro-grid and Smart Distribution System, Automation of Electric Power Systems, Vol.34 (2010), p.10-14.

[3] Shengqing Li, Lilin Zeng, Xiaodong Luo, Electric Power System's Reactive Optimization based on Multiple-objective Decision-Making Evolutionary Algorithm, Low Voltage Apparatus, Vol. 4 (2013), p.45-49.

[4] Shuangxi Zhou, Electric Power System's Voltage Stability and Control, Beijing: China Electric Power Press, 2004.

[5] Jiguang Liu, Haiyang Wang, Lijun Zhong, Hopf Bifurcation Control Simulation of Voltage Stability in Wind Power System, Jilin University Journal (Science Edition), Vol.1 (2013), p.111-115. 